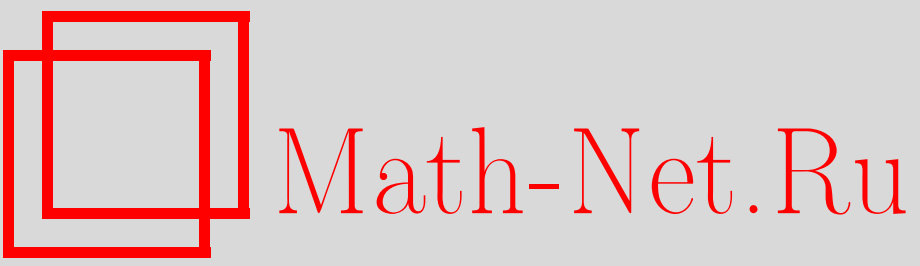

В. Ф. Ковалев, Д. В. Ширков, Функциональная автомодельность и ренормгрупповая симметрия в математической физике, ТМФ, 1999, том 121, номер 1, 66-88

DOI: https://doi.org/10.4213/tmf798

Использование Общероссийского математического портала Math-Net.Ru подразумевает, что вы прочитали и согласны с пользовательским соглашением

http://www.mathnet.ru/rus/agreement

Параметры загрузки:

IP: 18.234 .197 .8

26 апреля 2023 г., 15:46:38 


\author{
ТЕОРЕТИЧЕСКАЯ \\ И МАТЕМАТИЧЕСКАЯ \\ ФИЗИКА \\ Том 121, № 1 \\ октябрь, 1999
}

(C) 1999 г

В. Ф. Ковалев ${ }^{*}$, Д.В. Ширков ${ }^{\dagger}$

\title{
ФУНКЦИОНАЛЬНАЯ АВТОМОДЕЛЬНОСТЬ И РЕНОРМГРУППОВАЯ СИММЕТРИЯ В МАТЕМАТИЧЕСКОЙ ФИЗИКЕ
}

Представлен обзор развития за последние 10 лет исследований, связанных с использованием понятий функциональной автомодельности и ренормгруппы Боголюбова в краевых задачах математической физики. Основное достижение - построение регулярного алгоритма нахождения симметрий ренормгруппового типа с помощью современной теории групп Ли преобразований.

\section{1. ВВЕДЕНИЕ}

Понятие функциональной автомодельности $(\Phi А)$ было введено в математическую физику одним из авторов в начале восьмидесятых годов [1] (см. также работы $[2,3]$ ). Основанием для этого стало осознание того факта, что в основе широкого круга физических задач, анализируемых с помощью метода ренормализационной группы (РГ), лежит единое свойство инвариантности решений этих задач относительно групповых преобразований, затрагивающих не только естественные независимые переменные задачи, но также параметры граничных условий, задаваемых в некоторой “опорной” точке. При этом РГ-преобразование соответствует репараметризации некоторого данного решения при переходе к другой опорной точке путем изменения (сдвига или растяжения) некоторой независимой (координатной) переменной и одновременного функционального преобразования (граничных) характеристик искомых функций. Указанные “функции преобразования" подчиняются групповым функциональным уравнениям.

Оговоримся для ясности, что здесь имеются в виду так назьваемые перенормировочные преобразования (преобразования Дайсона) квантовой теории поля (КТП), которые образуют непрерывную однопараметрическую группу, т.е. группу Ли преобразований в обычной математической терминологии. Эта группа была открыта [4] и применена [5] для анализа сингулярностей КТП в первой половине 50-х годов. Для того чтобы отличать эту группу от распространенной в теоретической физике приближенной РГ, введенной в начале 70-х годов Вильсоном [6] для анализа критических явлений

\footnotetext{
${ }^{*}$ Институт математического моделирования РАН, Москва, Россия. E-mail: kovalev@imamod.ru

† Лаборатория теоретической физики им. Н. Н. Боголюбова, ОИЯИ, Дубна, Россия. E-mail: shirkovd@thsun1.jinr.ru
} 
в задачах статистической физики, мы будем называть ее квантово-полевой или боголюбовской РГ.

Такое положение соответствовало представлению о преобразованиях РГ, рассматриваемых в контексте КТП. Распространение метода РГ на классические задачи математической физики, которое вначале происходило путем трансформации и применения уже разработанных приемов в КТП и статистической физике, в середине восьмидесятых получило новый импульс. Стимулом для этого послужило, с одной стороны, уже упомянутое выше представление о $\Phi$ А физических задач, позволяющее выделить характерные переменные задачи (независимые и динамические переменные, параметры и краевые данные) и указать возможньй характер преобразований, который оставляет инвариантным решение задачи, а с другой стороны, те выгоды в математическом описании исследуемых систем, которые достигались использованием моделей, базируюшихся лишь на дифференциальных и интегродифференциальных уравнениях. Эти выгоды проявились в том, что для построения соответствующих преобразований ренормгруппового типа оказалось возможным использовать регулярные методы нахождения симметрий, применяемые в групповом анализе дифференциальных уравнений (ДУ), тем более что за последние несколько десятков лет, прошедших с момента возникновения метода РГ, в теоретической физике и в области группового анализа достигнут весьма существенный прогресс.

При сохранении основной сущности понятия $\Phi$ А как свойства, характеризующего инвариантность решения физической задачи относительно преобразований особого класса, переход к краевым задачам математической физики дал возможность расширить математическое представление этого свойства. Первоначальное представление о преобразованиях $\Phi$ А как о точечных преобразованиях, связанных с преобразованием независимых переменных, в последнее время дополнено и охватывает не только точечные преобразования, но и контактные преобразования, преобразования в виде формальных рядов и т.п. Поскольку для математических моделей, основанных на ДУ, удобно использовать в групповом анализе язык бесконечно малых преобразований, то и $\Phi$ А можно формулировать с помощью инфинитезимальных операторов, задающих соответствующую ренормгрупповую симметрию (РГС). При этом сами операторы возникают на основе регулярной процедуры построения РГС, а условие $\Phi$ А используется как составной элемент этой процедуры.

Предметом нашего обзора являются описание эволюции понятия $\Phi$ А, связанного с развитием представления о РГС в математической физике, и последовательное изложение результатов, полученных на основе ее использования.

Статья построена по следующей схеме. В разделе 2, во-первых, вводятся РГ-преобразования, как инфинитезимальные, так и конечные. Во-вторых, устанавливается их связь с известным в математической физике понятием степенного самоподобия (автомодельности) и вводится понятие $\Phi$ А. Наконец, излагается способ регулярного построения РГС в математической физике и выделено значение свойства $\Phi$, которое является составным элементом этого способа. При этом отмечена трансформация понятия $\Phi$ А при переходе от боголюбовской РГ к моделям математической физики.

В третьем разделе рассмотрены случаи преобразований $\Phi А$, которые по своим свойствам близки к квантово-полевым в том смысле, что они реализуются как группы однопараметрических преобразований по некоторым независимым переменным. Указаны при- 
меры, когда группа расширяется и порождает преобразования $\Phi$ А с операторами, образуюшими алгебру конечной размерности. Показано, что аналогия между обсуждаемой в этом разделе математической моделью в виде обыкновенного ДУ (ОДУ) или системы таких уравнений и совпадающими с ними по виду уравнениями Ли, возникающими в квантово-полевых приложениях метода РГ, позволяет использовать развитый формализм и для этих последних.

В разделе 4 приводятся различные примеры условий $\Phi$ А как в виде ОДУ первого порядка, так и в виде дифференциальных соотношений более высокого порядка. Обсуждается развитие представления о преобразованиях $\Phi$ А, которые наряду с традиционными формами в виде алгебраических соотношений могут также задаваться преобразованиями в виде формального бесконечного ряда. Такой вид преобразований $\Phi$ А возникает при задании РГС в виде группы Ли-Беклунда ${ }^{1)}$, а условие $\Phi$ А реализуется в виде условия инвариантности конкретного частного решения краевой задачи относительно указанных преобразований.

Анализу условий $\Phi$ А для систем с малыми параметрами посвяшен пятый раздел. Отмечается, что использование этих условий позволяет находить приближенные РГС и применять их к решению задач с произвольными краевыми условиями. Приводятся примеры приближенных РГС для нескольких задач нелинейной физики.

Последний, шестой раздел работы посвяшен обсуждению перспектив развития понятия $\Phi А$ и его использования для построения РГС в широком спектре задач математической физики.

\section{2. КВАНТОВО-ПОЛЕВАЯ РЕНОРМГРУППА И УСЛОВИЕ ФУНКЦИОНАЛЬНОЙ АВТОМОДЕЛЬНОСТИ}

2.1. Простые преобразования боголюбовской ренормгруппы. Для иллюстрации понятия $\Phi$ А рассмотрим простейшее РГ-преобразование, которое представляет собой одновременное однопараметрическое преобразование одной из независимых переменных задачи (например, координаты) $\ell$ и некоторой характеристики решения $g$,

$$
T(\lambda):\left\{\ell \rightarrow \ell^{\prime}=\ell-\lambda, g \rightarrow g^{\prime}=G(\lambda, g)\right\}, \quad G(0, g)=g .
$$

Функция $G(\ell, g)$ удовлетворяет функциональному уравнению

$$
G(\ell+\lambda, g)=G(\ell, G(\lambda, g))
$$

которое отвечает групповому закону композиции операторов преобразований

$$
T\left(\lambda_{1}\right) T\left(\lambda_{2}\right)=T\left(\lambda_{1}+\lambda_{2}\right)
$$

Оператор преобразования (1) иногда полезно представить в явной форме, записав преобразование некоторой функции $F(\ell, g)$ в виде

$$
T(\lambda) F(\ell, g)=e^{-\lambda R} F(\ell, g)
$$

\footnotetext{
1) Обобщенная группа Ли преобразований - группа Ли-Беклунда - включает в число групповых переменных производные от искомых функций по независимым переменным.
} 
с помощью инфинитезимального оператора РГС (или РГ-оператора)

$$
R=\partial_{\ell}-\beta(g) \partial_{g}, \quad \beta(g)=\left.\frac{\partial G(\lambda ; g)}{\partial \lambda}\right|_{\lambda=0}
$$

координата которого $\beta(g)$ определена как производная функции $G$ в точке $\lambda=0$. Оператор $R$ удобен для представления РГ-преобразований в инфинитезимальной форме. В то же время из "конечного" представления (3) явно видна групповая природа оператора $T(\lambda)=e^{-\lambda R}$.

условие

$$
R I(\ell, g) \equiv \partial_{\ell} I-\beta(g) \partial_{g} I=0
$$

определяет инвариант РГ-преобразования, который в данном случае представим в виде функции одного аргумента $I(\ell, g)=\tilde{I}(G(\ell, g)) \equiv I(0, G(\ell, g))$, поскольку функция преобразования $G(\ell, g)$ согласно уравнению (2) сама является инвариантом, тогда как условие

$$
R C(\ell, g)=\varphi(g) C(\ell, g)
$$

отвечает коварианту, т.е. величине, преобразуюшейся по представлению группы $Ф А$ (РГ). Коварианты играют важную роль в квантово-полевых применениях РГ.

Заметим, что представление РГ-преобразования с помощью инфинитезимального оператора $R$ эквивалентно записи в виде конечного преобразования (3). Функциональное уравнение (2) следует из характеристического уравнения для оператора $R$, а при заданной $\beta$-функции можно построить и явное выражение для $G$, решая соответствующие уравнения Ли для оператора (4)

$$
-d \ell^{\prime}=\frac{d g^{\prime}}{\beta\left(g^{\prime}\right)}=d \lambda
$$

с начальными условиями $\left.\ell^{\prime}\right|_{\lambda=0}=\ell,\left.g^{\prime}\right|_{\lambda=0}=g$.

Для дальнейшего сушественно, что условие инвариантности функции $I$ под действием РГ-преобразований, записанное в виде уравнения (5), совпадает с условием равенства нулю координаты $\varkappa$ оператора (4), представленного в канонической форме

$$
\bar{R}=\varkappa \partial_{I}, \quad \varkappa \equiv I_{\ell}-\beta(g) I_{g}=0 .
$$

Это условие следует рассматривать на частном решении $I(\ell, g)$ краевой задачи.

В КТП естественными являются “экспоненцированные” переменные

$$
x=e^{\ell}, \quad a=e^{\lambda}, \quad \bar{g}(a, g)=G(\lambda, g) .
$$

В этом представлении групповое преобразование $T(\lambda)=T_{a}$ записывается в виде

$$
T_{a}:\left\{x^{\prime}=x / a, g^{\prime}=\bar{g}(a, g)\right\}, \quad \bar{g}(1, g)=g,
$$

а также

$$
\bar{g}(x, g)=\bar{g}(x / a, \bar{g}(a, g)), \quad T_{a} \cdot T_{b}=T_{a b}
$$


Функциональное уравнение (10) и преобразование (9) возникают, например, в КТП с одной константой связи в безмассовом случае. При этом безразмерная координата $x=Q^{2} / \mu^{2}$ имеет смысл отношения квадрата переданного 4-импульса $Q$ к квадрату "нормировочного" импульса $\mu, g$ является константой связи, а инвариант $\bar{g}$ называется инвариантной (или эффективной) функцией связи. Более сложные примеры РГ-преобразований в КТП получают обобщением преобразований типа (9) путем "размножения" характеристик решения $g \rightarrow\{g\}=\left(g_{1}, g_{2}, \ldots, g_{k}\right)$, а также введением некоторых дополнительных параметров в функции $\bar{g}_{i}(x,\{g\})($ см. $[7, \S 49])$.

Так, например, в КТП-модели с двумя константами связи $g_{1}=g$ и $g_{2}=h$ и одной массой частищы $m$ преобразование $T_{a}$ принимает вид

$$
\begin{gathered}
T_{a}: \quad\left\{x^{\prime}=x / a, y^{\prime}=y / a, g^{\prime}=\bar{g}(a, y ; g, h), h^{\prime}=\bar{h}(a, y ; g, h)\right\} \\
\bar{g}(1, y ; g, h)=g, \quad \bar{h}(1, y ; g, h)=h, \quad y=Q^{2} / \mu^{2}
\end{gathered}
$$

Связанные с инфинитезимальным оператором

$$
\begin{gathered}
R=x \partial_{x}+y \partial_{y}-\beta_{1}(y ; g, h) \partial_{g}-\beta_{2}(y ; g, h) \partial_{h}, \\
\beta_{1}(y ; g, h)=\left.\frac{\partial \bar{g}(\xi, y ; g, h)}{\partial \xi}\right|_{\xi=1}, \quad \beta_{2}(y ; g, h)=\left.\frac{\partial \bar{h}(\xi, y ; g, h)}{\partial \xi}\right|_{\xi=1},
\end{gathered}
$$

уравнения характеристик в случае нескольких констант связи образуют систему ДУ первого порядка

$$
x \frac{\partial \bar{g}(x, y ; g, h)}{\partial x}=\beta_{1}\left(\frac{y}{x} ; \bar{g}, \bar{h}\right), \quad x \frac{\partial \bar{h}(x, y ; g, h)}{\partial x}=\beta_{2}\left(\frac{y}{x} ; \bar{g}, \bar{h}\right),
$$

однако в основе всех этих однопараметрических преобразований лежит преобразование одного независимого аргумента $x$.

2.2. Самоподобие и функциональная автомодельность. В частном случае, когда функция $\bar{g}$ линейна по второму аргументу, $G \sim g$, решение уравнения (10) имеет степенную зависимость от аргумента $x$, т.е. $\bar{g}(x, g)=g x^{k}$, где $k$ - произвольное число, и преобразования (9) принимают вид преобразований степенного самоподобия (так называемых автомодельных преобразований)

$$
P_{a}:\left\{x^{\prime}=x / a, \quad g^{\prime}=g a^{k}\right\}
$$

широко используемых при исследовании задач динамики газа и жидкости. С этих позиций преобразования $(9),(10)$ в общем случае произвольных $G, \bar{g}$ могут рассматриваться как функциональное обобшение $g x^{k} \rightarrow \bar{g}(x, g)$ обычных автомодельных преобразований, что и дало основание назвать их преобразованиями функииональной автомодельности [1]; этот термин является синонимом преобразований РГ.

Из сказанного следует, что в рамках введенных выше РГ-преобразований понятие $\Phi$ А отражает групповую природу функциональных соотношений. Универсальность формулировки $\Phi$ А в квантово-полевых и иных моделях классической физики базируется на 
едином преобразовании растяжения шкалы и функциональном преобразовании величины характеристики решения $g_{\mu}=\bar{g}(\mu, g)$. При этом различные реализации РГ отличаются лишь конкретным видом функции $\beta(g)$, которая в каждом случае вычисляется с помошью приближенного решения, обычно полученного по теории возмушений (ТВ). Таким образом, до недавнего времени основная роль $\Phi$ А заключалась в апостериорной констатации того факта, что исследуемая система допускает функциональные преобразования, имеющие групповую природу. Сами же эти групповые преобразования устанавливались на основе дополнительных представлений о характере решения системы [8].

2.3. Метод построения РГС. Ситуация существенным образом изменилась при переходе к исследованию краевых задач в математической физике. Здесь свойство $\Phi$ А исследуемого решения используется для отыскания функциональных соотношений, отражаюших групповую природу решения, или, если перейти на язык бесконечно малых, для построения симметрий $\mathrm{P} \Gamma(=\Phi А)$ типа. Для современных моделей математической физики РГС находится регулярным способом путем использования схемы [9], в которую естественным образом входят понятие $\Phi$ А и его формулировка в виде условия инвариантности частного решения краевой задачи.

Для того чтобы указать роль и место приложения условия $\Phi$ А в построении РГС, напомним основные моменты этой схемы, которые можно сформулировать в виде последовательности четырех основных шагов.

Первый шаг состоит в построении специального ренормгруппового многообразия (дифференциального, интегродифференциального, ...), отличающегося, вообще говоря, от многообразия, которое задает система исходных уравнений, описывающих исследуемую физическую систему. Второй шаг заключается в отыскании допускаемой РГ-многообразием максимально широкой группы преобразований $\mathcal{G}$. Третьим шагом в процессе построения РГС является сужение найденной группы $\mathcal{G}$ на решении краевой задачи (точном или приближенном). Возникаюшая при этом группа преобразований, которую мы и именуем РГ, представляется набором инфинитезимальных операторов $R_{i}$, каж дый из которых в своем инвариантном многообразии содержит решение краевой задачи. На последнем, четвертом шаге инфинитезимальные РГ-операторы $R_{i}$ используются для построения конечных преобразований группы и получения аналитического выражения для решения краевой задачи.

Первый шаг в описанной схеме построения РГС имеет фундаментальное значение и в зависимости от структуры математической модели и от вида граничных условий может быть реализован различными способами [9]: искомое РГ-многообразие можно получить, расширяя список групповых переменных добавлением параметров, входяших в исходные уравнения и краевые данные, или включая в этот список производные от имеюшихся зависимых переменных и нелокальные переменные. Можно также расширять число исходных уравнений, записывая краевые данные в виде уравнений погружения или в виде дополнительных дифференциальных связей. В ряде случаев расширения группы можно достичь частичным упрошением исходных уравнений за счет малых параметров.

Вычисляемая на втором шаге с использованием алгоритмов современного группового анализа [10] максимальная группа $\mathcal{G}$ для РГ-многообразия еше не является РГ, поскольку не связана с конкретным частным решением. 
Для построения последней выполняется третий шаг, а именно проводится процедура сужения найденной групшы $\mathcal{G}$ на частном точном или приближенном решении искомой краевой задачи. Математически процедура сужения сопровождается "комбинированием" канонических координат операторов группы, допускаемой РГ-многообразием. Условие обрашения в нуль суммы этих координат на решении краевой задачи (условие инвариантности относительно оператора РГС, аналогичное условию (8)) приводит к набору алгебраических равенств, связываюших между собой координаты различных операторов и порождаюших таким образом искомые РГС. С физической точки зрения в каждом конкретном случае проверка этого условия и есть реализация принципа $\Phi$ А, которьй таким образом устанавливает инвариантность частного решения относительно преобразований РГ. С другой стороны, этот шаг существенным образом использует знание о решении рассматриваемой задачи по ТВ, реализуя общий принцип метода РГ, нацеленного на построение улучшенного (по сравнению с ТВ) решения. Следует отметить, что параметром ТВ в такой схеме может выступать любой из подходящих с физической точки зрения параметров или набор этих параметров.

Проверка условия $\Phi$ А в сформулированной выше схеме построения РГС отличается от процедуры проверки условия инвариантности анализируемых решений относительно итерационной последовательности масштабных преобразований, которые используют при анализе поведения физической системы в теории критических явлений [6] и также называют РГ-преобразованиями, хотя в общем случае они не образуют группу (в отличие от моделей КТП, где сушествование группового свойства является итогом специального доказательства $[7,8])$. В последнее время эта идеология (здесь мы называем ее вильсоновской РГ) получила широкое распространение в математической физике, где она использовалась для проведения асимптотического анализа поведения решений ДУ $[11,12]$ и для построения огибаюшей семейства решений [13]. В рамках вильсоновской РГ в приложении к задачам математической физики алгоритм улучшения решений по ТВ, содержащих сингулярности, реализуется путем введения некоторых дополнительных параметров в решения, устранения с их помошью особенностей и наложения условия независимости решений от способа введения этих параметров [11]. Вопрос о соответствии таких построений группе преобразований искомого решения краевой задачи и возможности его получения остается открытым, хотя в отдельных случаях этот алгоритм и приводит к успеху.

Отражением РГ-симметрии в математической физике является набор операторов РГС. Таким образом, как и в классическом групповом анализе ДУ, достаточно рассматривать бесконечно малые преобразования и характеризовать их алгеброй инфинитезимальных операторов. Отметим, что в отличие от типичной для квантово-полевых моделей ситуации, когда имеется лишь один оператор, РГ-операторы в математической физике образуют алгебру, состояшую, вообше говоря, более чем из одного оператора. Как размерность этой алгебры, так и способ ее построения существенным образом зависят от используемой математической модели и характера задания граничных условий.

При этом в роли группы симметрии, сужаемой на решении краевой задачи, может быть использована не только точечная группа Ли, но и группа Ли-Беклунда, приближенная группа преобразований, группа нелокальных симметрий, группа нелиевских симметрий и т.д. [9]. Ниже мы рассмотрим реализацию условий $\Phi$ А и различные формы записи преобразований $\Phi$ в вяде конкретных задач, использующих РГС. 


\section{3. АНАЛИЗ ФУНКЦИОНАЛЬНОЙ АВТОМОДЕЛЬНОСТИ СИСТЕМ, БЛИЗКИХ К КВАНТОВО-ПОЛЕВЫМ}

Начнем с иллюстрации преобразований $Ф$ А для систем, описываемых ОДУ, и для простоты рассмотрим краевую задачу для функции $u(t)$, удовлетворяюшей ОДУ первого порядка $[14,15,9]$ с явно входящими в уравнение параметрами $b^{k}(k=1,2, \ldots)$,

$$
\begin{gathered}
u_{t}=f\left(t, u, b^{k}\right), \\
u(\tau)=x .
\end{gathered}
$$

Этот пример имеет не только методический характер, но и дает представление о возможных направлениях развития понятия $\Phi$ А, в частности в приложении к КТП, где подобные уравнения возникают в методе РГ на этапе анализа системы ДУ для инвариантных функций связи.

Построение РГС для краевой задачи (14), (15) в рамках сформулированного выше алгоритма [9] производится путем добавления к (14) уравнения погружения, которое имеет вид линейного ДУ с частными производными первого порядка:

$$
u_{\tau}+f\left(\tau, x, b^{k}\right) u_{x}=0
$$

Объединение уравнений (14), (16) в единую систему дает исходное РГ-многообразие в пространстве всех групповых переменных $\left\{u, t, \tau, x, b^{k}\right\}$, включающих, помимо зависимых и независимых переменных и параметров, присутствуюших в исходном уравнении (14), также параметры $\tau, x$, входящие в краевые данные.

Вычисление допускаемой многообразием (14), (16) группы симметрии $\mathcal{G}$ приводит к различным результатам в зависимости от вида функции $f$. В практически интересном (ультрафиолетовом пределе для моделей КТП) случае отсутствия явной зависимости этой функции от времени $t$ и при наличии только трех параметров $b^{1} \equiv a, b^{2} \equiv b$ и $b^{3} \equiv c$ в исходном уравнении, т.е. при $f=f(u, a, b, c)$, допускаемая группа $\mathcal{G}$ задается оператором с семью вкладами

$$
X=\sum_{i=1}^{7} \alpha_{i} X_{i}
$$

Первые две функции $\alpha_{1}$ и $\alpha_{2}$ являются произвольными функциями всех семи групповых переменных $\{t, \tau, x, a, b, c, u\}$, а остальные произвольным образом зависят от параметров $a, b, c$ и комбинаций $\tilde{t}=t-\langle 1 / f(u)\rangle$ и $\tilde{\tau}=\tau-\langle 1 / f(x)\rangle$. Угловые скобки обозначают интеграл соответственно по переменной $u$ или $x$. Явные выражения для трех из входящих в (17) операторов имеют вид

$$
\begin{gathered}
X_{1}=\partial_{t}+f(u) \partial_{u}, \quad X_{3}=f(u) \partial_{u} \\
X_{5}=f(x)\left\langle\frac{f_{a}(x)}{f^{2}(x)}\right\rangle \partial_{x}+f(u)\left\langle\frac{f_{a}(u)}{f^{2}(u)}\right\rangle \partial_{u}+\partial_{a}
\end{gathered}
$$

Остальные операторы получаются из них подстановками: $X_{2}$ и $X_{4}$ следуют из $X_{1}$ и $X_{3}$ при заменах $t \rightarrow \tau$ и $u \rightarrow x$, а $X_{6}$ и $X_{7}-$ из $X_{5}$ при замене производной $\partial_{a}$ на $\partial_{b}$ и $\partial_{c}$. 
Операция сужения групш $\mathcal{G}$ заключается в проверке условия $\Phi$ А, аналогичного равенству $\varkappa=0$ в (8), т.е. в проверке инвариантности решения относительно РГ-преобразований, или, иными словами, условия обрашения в нуль координаты канонического оператора $X$ на решении начальной задачи $u=U(t, x, \tau, a, b, c)$. В результате вьполнения процедуры сужения, допускаемой многообразием $(14),(16)$ группы $\mathcal{G}$ на любом доступном (приближенном) решении задачи (14), (15), возникают некоторые "суженные симметрии", которые мы условимся именовать ${ }^{2)}$ РГС.

В зависимости от конкретной постановки задачи в качестве функции $U$ можно использовать тот или иной отрезок ряда ТВ. Например, для функции $f$ в виде полинома

$$
f=a u^{2}+b u^{3}+c u^{4}
$$

можно обсуждать ряды ТВ по переменным $(t-\tau)$, а также по параметрам $a, b$ или $c$. Пример операции сужения группы на решении с использованием ТВ по $a$ при $b=c=0$ рассматривался в [14,9], а по параметру $b$ при $a=1, c=0-$ в [9]. Ниже приводятся два возможных оператора РГС, возникающих при таком сужении $[14,9]$ :

$$
\begin{aligned}
& R_{1}=x^{2} \tau \partial_{x}+\partial_{a}+u^{2} t \partial_{u}, \quad b=c=0, \\
& R_{2}=\left(x^{2}(1+b x) \tau+x\right) \partial_{x}+\left(u^{2}(1+b u) t+u\right) \partial_{u}-b \partial_{b}, \quad a=1, \quad c=0 .
\end{aligned}
$$

Для получения решения начальной задачи следует теперь записать условие его инвариантности относительно оператора РГС - условие $\Phi$ А, которое имеет вид линейного ДУ в частных производных первого порядка. Например, для оператора $R_{1}$

$$
t u^{2}-x^{2} \tau u_{x}-u_{a}=0 .
$$

Решение уравнений характеристик для этого уравнения - уравнений Ли - дает искомое решение начальной задачи при $b=c=0$. Отметим, что условие $\Phi$ А вида (20) используется дважды: как при построении операторов РГС, когда вместо $u$ в (20) подставляется приближенное решение $U$, так и при нахождении с помошью этих операторов решения краевой задачи.

В обоих примерах построение ТВ не представляет особой сложности и приводит либо к степенной зависимости, либо к комбинации степенной, обратно пропорциональной и логарифмической зависимостей от начальных значений. При этом процедура построения ряда ТВ по степеням $b$ для получения второго оператора $R_{2}$ из (19) использует в качестве невозмушенного состояния решение начальной задачи при $a=1$, которое возникало в результате применения первого оператора $R_{1}$, т.е. в приближении сильной (по параметру $a$ ) нелинейности. Таким образом, результат улучшения ТВ с использованием оператора $R_{2}$ можно рассматривать как результат последовательного улучшения ТВ для краевой задачи с функцией $f=a u^{2}+b u^{3}$ сперва по параметру $a$, а затем по параметру $b$ с помощью однопараметрических групп $R_{1}$ и $R_{2}$.

Такая процедура улучшения ТВ последовательно по нескольким входящим в модель параметрам не всегда реализуема, например, из-за невозможности построить “нелинейное" невозмушенное решение. Для обсуждаемой здесь функции $f$ в виде полинома по

\footnotetext{
${ }^{2)}$ Отметим, что некоторые из операторов $R$ таким образом определенных РГС могут наследоваться от симметрии уравнений.
} 
степеням $u$ проблема построения ряда ТВ по параметру $c$ при фиксированных $a, b$ не является столь же простой, как построение ТВ при $c=0$, поскольку невозмущенное состояние определено в этом случае с помощю функции Ламберта [16], не имеющей простого аналитического представления (см. также [17]).

Поэтому интересен вопрос о построении такой РГС, которая порождала бы групповые преобразования одновременно по нескольким параметрам и давала возможность улучшать ТВ, для которой нахождение невозмушенного решения не представляло бы затруднений. По сушеству, речь идет о построении двухпараметрической РГ. Подробное обсуждение этого вопроса составит предмет отдельных будущих исследований, а здесь для иллюстрации приводится лишь один пример такой РГ:

$$
\begin{aligned}
& R_{3}=-\left\langle\frac{f_{b}(u)}{f^{2}(u)}\right\rangle \partial_{t}-\left\langle\frac{f_{b}(x)}{f^{2}(x)}\right\rangle \partial_{\tau}+\partial_{b} \\
& R_{4}=-\left\langle\frac{f_{c}(u)}{f^{2}(u)}\right\rangle \partial_{t}-\left\langle\frac{f_{c}(x)}{f^{2}(x)}\right\rangle \partial_{\tau}+\partial_{c}
\end{aligned}
$$

Нетрудно убедиться, что проверка условий $\Phi$ А, т.е. проведение процедуры сужения группы $\mathcal{G}$ на пертурбативном решении одновременно по двум параметрам $b \rightarrow 0$ и $c \rightarrow 0$ не вызывает технических затруднений, а использование конечных преобразований, порождаемых операторами $R_{3}$ и $R_{4}$, образуюшими двумерную алгебру, приводит к искомому решению задачи (14), (15). Таким образом, использование двухпараметрической РГ позволяет преодолеть математические сложности, возникающие при использовании однопараметрических РГ.

Сделаем несколько замечаний в заключение этого раздела. Выше мы продемонстрировали использование условия $\Phi$ А в построении РГС, улучшаюших ТВ по входяшим в уравнение параметрам $a, b$ и $c$, хотя, конечно, соответствующие операторы не исчерпывают всего бесконечного набора РГС, который характеризуется континуальным произволом координат РГ-операторов $[15,14,9]$. Аналогичным образом можно находить операторы РГС, улучшающие ТВ по подходящей комбинации динамических переменных и начальных параметров (например, по разности $(t-\tau)$ ). Примеры таких РГС будут приведены в следующих разделах. Здесь мы только отметим очевидную аналогию условия $\Phi$ А в виде равенства (8) и условия $\Phi$ А (20), возникшего для краевой задачи (14), (15). Рассмотренные в этом разделе примеры РГС и связанные с ними условия $\Phi$ А касались единственного ОДУ. Похожие построения можно выполнить и для систем ОДУ, содержаших несколько параметров. Несколько примеров записи уравнений погружения для этих систем, используемых для нахождения РГС, приведены в [14].

\section{4. УСЛОВИЯ ФУНКЦИОНАЛЬНОЙ АВТОМОДЕЛЬНОСТИ ДЛЯ ДУ С ЧАСТНЫМИ ПРОИЗВОДНЫМИ}

Обратимся к примерам систем, использующих в качестве математических моделей ДУ или системы уравнений в частных производных. В отличие от предыдущего раздела, где появление в условиях $\Phi$ А частных производных первого порядка было связано с преобразованием параметров в исходных уравнениях, а также с уравнениями погружения, т.е. с учетом краевых данных, здесь мы продемонстрируем краевые задачи, для 
которых условия ФА имеют вид системы ДУ (связей), содержаших частные производные более высокого порядка по независимым переменным. При этом оказывается, что дифференциальная формулировка условий $\Phi$ А позволяет построить искомое решение задачи, в то время как процедура получения конечных преобразований $\Phi$ А ведет к формальным степенным рядам [18, т. 3, гл. 1]. Кроме того, будет приведен пример более простого, чем в разделе 3, условия $\Phi$ А в виде ОДУ, решение которого также восстанавливает решение краевой задачи по заданной ТВ.

4.1. Краевая задача для уравнения Бюргерса. Наглядный пример, иллюстрируюший разнообразие формулировок условия ФА для систем, основанных на ДУ в частных производных, дает краевая задача для модифицированного уравнения Бюргерса

$$
\begin{gathered}
u_{t}-a u_{x}^{2}-\nu u_{x x}=0, \\
u(0, x)=f(x)
\end{gathered}
$$

с параметрами нелинейности $a$ и диссипации $\nu$. Непрерывная точечная группа симметрии, допускаемая многообразием (22), представляется набором из девяти операторов. Шесть из них описывают симметрию уравнения и обсуждались в литератуpe (см., например, [18, т. 1, гл. 1, с. 183]). Они отвечают проективному преобразованию, преобразованию растяжения в плоскости $(t, x)$, переносам по осям $t, x$ и $u$ и преобразованию Галилея. Седьмой - оператор бесконечного абелева идеала группы $X_{\infty}=\alpha \exp (-a u / \nu) \partial_{u}$, координата которого имеет континуальный произвол в виде функции $\alpha(t, x, a, \nu)$ четырех групповых переменных, ограниченный линейным параболическим уравнением

$$
\alpha_{t}-\nu \alpha_{x x}=0
$$

совпадаюшим с линейной частью $(a=0)$ исходного уравнения $(22)$. Наконец, еше два оператора возникают при вовлечении параметров уравнения как независимых переменных в групповые преобразования и соответствуют преобразованию растяжения $a$ и $\nu[19]$.

Процедура сужения группы, допускаемой многообразием $(22)$, на решении $u=U(t, x$, $a, \nu)$ начальной задачи - проверка условия $\Phi$ А - дает алгебраическое соотношение, выражающее координату оператора бесконечномерной подгруппы (функцию $\alpha$ ) через координаты остальных восьми операторов в произвольный момент времени $t$, в том числе и при $t=0$, когда это решение $U(0, x, a, \nu)=f(x)$ известно из начального условия (23). Использование стандартного представления для решения линейного уравнения для функции $\alpha$ с начальным значением $\alpha(0, x, a, \nu)$, полученным из условия $\Phi$ А, и подстановка этого представления в формулу, определяющую общий элемент алгебры Ли, дают искомые операторы РГС. Таким образом, РГС для начальной задачи (22), (23) получаются комбинированием симметрий восьмимерной алгебры с указанными выше операторами и симметрий бесконечномерной подалгебры с оператором $X_{\infty}$. Каждый из полученных восьми РГ-операторов (и их линейные комбинации с произвольными коэффициентами - функциями от $a$ и $\nu$ ) содержит решение начальной задачи $u=U(t, x, a, \nu)$ в инвариантном многообразии и позволяет находить групповые преобразования как самих переменных $\{t, x, a, \nu\}$, так и различных функционалов от решения (локальных и нелокальных). 
Ниже мы приведем два примера таких операторов РГС, позволяюших улучшить ТВ по соответствующему параметру. Первый из них служит для улучшения ТВ по параметру $a$, а второй - по независимой переменной $t$ :

$$
\begin{aligned}
& R_{5}=\partial_{a}+\frac{1}{a}\left(-u+\exp \left(-\frac{a u}{\nu}\right)\langle\langle f(x)\rangle\rangle\right) \partial_{u}, \\
& R_{6}=\partial_{t}+\exp \left(-\frac{a u}{\nu}\right)\left\langle\left\langle a f_{x}^{2}+\nu f_{x x}\right\rangle\right\rangle \partial_{u} .
\end{aligned}
$$

Двойными угловыми скобками обозначена интегральная свертка заключенной в них функции с фундаментальным решением $G(t, x, \nu)$ линейного уравнения для функции $\alpha$, умноженным на экспоненту от функции $f$ из граничного условия (23):

$$
\langle\langle F(x)\rangle\rangle \equiv \frac{1}{\sqrt{4 \pi \nu t}} \int_{-\infty}^{\infty} d y F(y) \exp \left(-\frac{(x-y)^{2}}{4 \nu t}+\frac{a f(y)}{\nu}\right) .
$$

Соответствуюшие РГ-операторам (24) и (25) условия инвариантности решения краевой задачи - условия $\Phi$ А - записываются в виде двух ОДУ первого порядка:

$$
\begin{aligned}
& -u_{a}-\frac{u}{a}+\frac{1}{a} \exp \left(-\frac{a u}{\nu}\right)\langle\langle f(x)\rangle\rangle=0, \\
& -u_{t}+\exp \left(-\frac{a u}{\nu}\right)\left\langle\left\langle a f_{x}^{2}+\nu f_{x x}\right\rangle\right\rangle=0 .
\end{aligned}
$$

Решением любого из них с надлежашими начальными условиями приближенные решения уравнения (22) продолжаются по параметрам $a$ или $t$ до единого точного решения $u=(\nu / a) \ln \langle\langle 1\rangle\rangle$, справедливого при всех значениях параметров $a$ и $t[19]$.

Обратим внимание на то, что условие $\Phi$ А (26) в данном примере краевой задачи для уравнения в частных производных оказалось записанным в виде ОДУ первого порядка. С формальной точки зрения оно проще, чем условия $\Phi$ А, сформулированные в разделе 3 для краевых задач для ОДУ, и отличается от соответствующих условий $\Phi$ А, возникающих в квантово-полевых моделях. С другой стороны, возврашаясь к формулировке краевых условий для ОДУ первого порядка на языке уравнений погружения, заметим, что условие (26) также можно рассматривать как своеобразное уравнение погружения краевой задачи $(22),(23)$ с параметром погружения $a$. В свою очередь, к краевой задаче $(26)$ с переменными $\{a, u\}$ и начальным условием $u=u_{0}(t, x, \nu)$ при $a=0$ также можно применить метод инвариантного погружения. Причем благодаря зависимости краевого значения $u_{0}$ от входящих в ОДУ (26) “параметров" $\{t, x, \nu\}$ соответствующее уравнение погружения будет интегродифференциальным [15]. Наряду с приведенными выше условиями $\Phi$ А в виде ОДУ первого порядка в наборе операторов РГС для краевой задачи $(22),(23)$ также присутствуют операторы, которые приводят к условиям $\Phi$ А как в виде ДУ в частных производных первого порядка, аналогичным рассмотренным ранее, так и просто к алгебраическим соотношениям [19]. Таким образом, в рамках решения одной краевой задачи условия $\Phi$ А могут принимать различную форму. 
4.2. Краевая задача для уравнений нелинейной оптики. Иногда оказывается невозможным по тем или иным причинам согласовать ТВ, построенную для краевой задачи, с условиями $\Phi$ А, если ограничиться только точечными симметриями. Мы приводим пример, для решения которого используется условие $\Phi$ А в виде системы ДУ второго порядка. Рассмотрим краевую задачу для системы уравнений из нелинейной оптики коллимированных волновых пучков

$$
\begin{gathered}
v_{t}+v v_{x}-\alpha n_{x}=0, \quad n_{t}+v n_{x}+n v_{x}=0, \\
v(0, x)=0, \quad n(0, x)=N(x) .
\end{gathered}
$$

Здесь безразмерные координаты $t$ и $x$ служат для описания пространственной эволюции производной эйконала пучка $v$ и безразмерной интенсивности $n$ в направлении вглубь среды и в поперечном направлении, параметр $\alpha$ определяет роль нелинейной рефракции. Преобразование годографа сводит (28) к системе линейных уравнений для переменных $\tau=n t$ и $\chi=x-v t$, где использовано обозначение $w=v / \alpha$,

$$
\begin{gathered}
\tau_{w}-n \chi_{n}=0, \quad \chi_{w}+\alpha \tau_{n}=0, \\
\tau(0, n)=0, \quad \chi(0, n)=H(n) .
\end{gathered}
$$

Формально к построению РГС и анализу условий $А$ для системы (30) можно подходить так же, как и в примере для уравнения Бюргерса. Как и в предыдущем случае, возникают конечномерная подалгебра операторов точечных симметрий и ее бесконечномерный идеал. Однако процедура сужения этого идеала с целью получения РГС [20, 21] приводит к необходимости решения системы линейных уравнений в частных производных, совпадающей с исходной (30), что характерно для линейных уравнений.

Поэтому ниже изложен иной способ построения РГС, основанный на использовании не точечных симметрий Ли, а симметрий Ли-Беклунда (см. [18, т. 3, гл. 1]), для которых также используются термины "высшие" и "обобшенные" симметрии. При этом искомые операторы РГС для краевой задачи (30), (31) удобно записывать в каноническом виде

$$
R=f \partial_{\tau}+g \partial_{\chi}
$$

с координатами $f$ и $g$, являющимися функциями в расширенном пространстве групповых переменных, включающем не только набор $(\tau, \chi, w, n, \alpha)$, но и производные произвольного конечного порядка от $\tau$ и $\chi$ по $n$ (возможно, также по $\alpha$ ). Способ построения симметрий Ли-Беклунда для краевой задачи (30), (31) детально обсуждался в [20]. Было показано, что эти симметрии задаются операторами вида (32) с координатами, которые являются линейными комбинациями от $\tau, \chi$, их первых и вторых производных с коэффициентами, зависящими от $w$ и $n$. Мы приводим выражения для координат только трех операторов:

$$
\begin{aligned}
& f_{1}=-\frac{\tau}{2}+n \tau_{n}+\frac{1}{2} n w \chi_{n}, \quad g_{1}=-\frac{\alpha w}{2} \tau_{n}+n \chi_{n} \\
& f_{2}=n \tau_{n}, \quad g_{2}=\chi_{n}+n \chi_{n n} \\
& f_{3}=\frac{1}{4} \tau-n \tau_{n}-\frac{5}{4} w n \chi_{n}+\left(-n+\frac{\alpha}{4} w^{2}\right) n \tau_{n n}-w n^{2} \chi_{n n} \\
& g_{3}=\frac{3}{4} \alpha w \tau_{n}-\left(2 n-\frac{\alpha}{4} w^{2}\right) \chi_{n}+\alpha w n \tau_{n n}+\left(-n+\frac{\alpha}{4} w^{2}\right) n \chi_{n n}
\end{aligned}
$$


Первый из них с координатами $f_{1}$ и $g_{1}$, линейными по первым производным, эквивалентен оператору точечной симметрии, остальные два являются операторами симметрии Ли-Беклунда второго порядка. Процедура сужения найденной для РГ-многообразия группы Ли-Беклунда заключается в проверке условия $\Phi$ А

$$
f=0, \quad g=0,
$$

которое должно выполняться на решении краевой задачи и согласовываться с краевыми условиями (30). Из вида координат $f$ и $g$ следует, что эти условия, будучи записанными в терминах краевой функции $H(n)$, должны подчиняться линейным ДУ с переменными коэффициентами. В частности, например, в случае $N(x)=\mathrm{ch}^{-2}(x)$ условие $\Phi$ А вьполняется для комбинаций $f=f_{1}+2\left(f_{2}+f_{3}\right), g=g_{1}+2\left(g_{2}+g_{3}\right)$, а искомый оператор РГС Ли-Беклунда имеет вид

$$
\begin{aligned}
R_{7}= & \left(2 n(1-n) \tau_{n n}-n \tau_{n}-2 n w\left(\chi_{n}+n \chi_{n n}\right)+\frac{\alpha}{2} n w^{2} \tau_{n}\right) \partial_{\tau}+ \\
& +\left(2 n(1-n) \chi_{n n}+(2-3 n) \chi_{n}+\right. \\
& \left.+\alpha w\left(2 n \tau_{n n}+\tau_{n}+\frac{w}{2}\left(n \chi_{n n}+\chi_{n}\right)\right)\right) \partial_{\chi} .
\end{aligned}
$$

Видно, что условие (34) для оператора $R_{7}$ приводит к ДУ второго порядка, что качественно отличает его от рассматриваемых ранее условий $\Phi$, которые имеют вид уравнений первого порядка. Именно, для последних конечные преобразования $\Phi$ А могут быть найдены в замкнутом виде как решения соответствуюших уравнений Ли, отражающих преобразования зависимых и независимых переменных и параметров. Для РГС с оператором Ли-Беклунда (35) решения уравнений Ли приводят к конечным преобразованиям, записанным в виде формальных степенных рядов. Однако это не означает, что использование условий $\Phi$ А в этом случае не дает практического результата. Именно, дифференциальная формулировка условий $\Phi$ А позволяет рассматривать соотношения (34) как дополнительные дифференциальные связи, которые должны быть учтены при отыскании решения краевой задачи. Совместное решение этих уравнений с исходными дает инвариантное относительно группы РГС (35) решение краевой задачи [20-22].

Подводя итог разделу, отметим, что условия $\Phi$ А в виде равенства нулю координат канонического РГ-оператора Ли-Беклунда, рассматриваемые как система дифференциальных связей, играют роль своеобразных уравнений погружения. Прямое использование условий $\Phi$ А приводит к задаче построения решения, инвариантного относительно найденного оператора РГС. Более содержательным примером использования условий $\Phi$ А является отыскание с их помощью операторов точечной РГС как групп, допускаемой исходными уравнениями и соотношениями $\Phi$ А [15]. При этом в приншипе можно обсуждать и операторы РГС, содержашие производные высшего порядка по входящим в уравнения параметрам.

\section{5. ФУНКЦИОНАЛЬНАЯ АВТОМОДЕЛЬНОСТЬ И РГС ДЛЯ СИСТЕМ С МАЛЫМИ ПАРАМЕТРАМИ}

Обратимся теперь к свойству ФА для систем, описываемых ДУ с малыми параметрами. Наличие в системе малого(ых) параметра(ов) $\alpha$ позволяет при построении РГС 
начинать с исследования упрошенной $(\alpha=0)$ модели, которая допускает более широкую группу симметрии по сравнению со случаем $\alpha \neq 0$. При учете малых по $\alpha$ вкладов эта симметрия наследуется исходной системой уравнений, что приводит к появлению дополнительных слагаемых в координатах операторов в виде поправок по степеням $\alpha$. Сужение полученной группы симметрии на точном или приближенном решении краевой задачи дает искомые РГС, которые также представляются операторами с координатами в виде бесконечных рядов по степеням малых параметров. Для краевых данных специального вида ряды обрываются, что приводит к точной РГС без ограничений на величину входяших параметров. Для краевых данных произвольного вида ограничение конечным числом слагаемых в бесконечных рядах при малых значениях параметров соответствует приближенной РГС. Ниже в этом разделе мы приводим два примера построения РГС и использования условия $\Phi$ А для систем, задаваемых уравнениями с малыми параметрами.

5.1. ФА в нелинейной теории плазмы. Рассмотрим краевую задачу для уравнений теории нелинейного взаимодействия лазерного излучения с плазмой [23]. Такое взаимодействие для $p$-поляризованной электромагнитной волны частоты $\omega$ с единственной $z$-компонентой магнитного поля, падающей из вакуума на неоднородную плазму, описывается системой нелинейных нестационарных уравнений (здесь она в явном виде не приводится) для шести скалярных функций: двух компонент вектора скорости электронов $v_{x}, v_{y}$, их плотности $n$, двух компонент электрического поля $E_{x}, E_{y}$ и $z$-компоненты магнитной индукции $B_{z}$, которые зависят от времени $t$ и двух координат $x$ и $y$.

Нелинейность этих уравнений оказывается сушественной в небольшой пространственной области вблизи плазменного резонанса (при $\omega_{L}^{2} \approx \omega^{2}$ ), где благодаря естественным параметрам малости, таким как плавная неоднородность плотности ионов $N(x)$ вдоль оси $x$ и малость углов падения $\vartheta$ лазерных лучей на плазму, сушествует иерархия компонент полей $p$-поляризованной световой волны в критической точке плазмы. Это позволяет при построении наследуемой точечной РГС выделить из полной системы шести исходных уравнений более простую систему двух одномерных нелинейных уравнений в частных производных для компонент скорости электронов $v$ и электрического поля $E$ вдоль градиента плотности [24, 23]

$$
\begin{gathered}
v_{t}+a v v_{x}-E=0, \quad E_{t}+a v E_{x}+\omega_{L}^{2} v=0, \\
\omega_{L}^{2} \equiv \frac{4 \pi\left|e e_{i}\right| N}{m} .
\end{gathered}
$$

Здесь функции $v$ и $E$ выражены в единицах безразмерного параметра нелинейности $a$, пропорционального значению магнитной индукции $B$ в критической точке на частоте лазера, координата $y$ входит в комбинации со временем $t \rightarrow t-(\vartheta y / c)$.

Система шести исходных уравнений допускает лишь конечную группу точечных преобразований, а именно группу транслящий по осям $t$ и $y$ при произвольной зависимости $N(x)$. При постоянной плотности ионов $N=$ const дополнительно появляются группа трансляций по оси $x$ и группа одновременных вращений в трех плоскостях, задаваемых координатами $\{x, y\}$ и соответствующими $x$ - и $y$-компонентами скорости электронов и электрического поля. В отличие от исходных уравнений система (36) допускает бесконечномерную группу точечных преобразований с оператором, представимым суммой 
трех вкладов:

$$
\begin{aligned}
X_{1} & =\mu_{1} Y, \quad X_{2}=\mu_{2} \partial_{x}+\frac{1}{a} Y\left(\mu_{2}\right) \partial_{v}+\frac{1}{a} Y^{2}\left(\mu_{2}\right) \partial_{E} \\
X_{3} & =\frac{1}{a} \mu_{3}\left(a \partial_{a}-v \partial_{v}-E \partial_{E}\right) \\
Y & =\partial_{t}+a v \partial_{x}+E \partial_{v}-\omega_{L}^{2} v \partial_{E}
\end{aligned}
$$

каждый из которых содержит произвольную функцию $\mu_{i}$ независимых и зависимых переменных и параметра $a$, причем на $\mu_{2}$ и $\mu_{3}$ наложены дифференшиальные связи

$$
Y^{3}\left(\mu_{2}\right)+Y\left(\omega_{L}^{2} \mu_{2}\right)=0, \quad Y\left(\mu_{3}\right)=0 .
$$

Для получения РГС группа точечных преобразований (37) сужается на приближенном по степеням параметра $a$ решении краевой задачи, которое строится таким образом, что нулевое приближение для функций $v, E$ определяется решением линеаризованной системы исходных уравнений с учетом соответствующих граничных условий (падение на плазму электромагнитной волны из вакуума) и выбранным профилем плотности $N(x)$ в области плазменного резонанса, а поправки к нему, пропорциональные $a$, возникают при линеаризации системы (36). Проверка условий $\Phi$ А для группы (37) на этом частном решении определяет выбор функций $\mu_{1}=0, \mu_{2}=-E / \omega^{2}, \mu_{3}=1$ и дает искомый оператор РГС

$$
R_{8}=X_{2}+X_{3}=-\frac{E}{\omega^{2}} \partial_{x}+\partial_{a}
$$

Условие инвариантности решения краевой задачи относительно РГ-оператора $R_{8}-$ условие $\Phi$ А - дается системой ДУ в частных производных первого порядка

$$
v_{a}-\frac{E}{\omega^{2}} v_{x}=0, \quad E_{a}-\frac{E}{\omega^{2}} E_{x}=0 .
$$

Решение уравнений характеристик для этой системы - уравнений Ли для оператора $R_{8}$ - восстанавливает ряд ТВ по параметру а до точного решения краевой задачи [23]

$$
\begin{gathered}
E=-\frac{(\omega L)^{2}}{\Delta}\left(q_{1} \sin \omega t+q_{2} \cos \omega t\right), \quad v=-\frac{\omega L^{2}}{\Delta}\left(q_{1} \cos \omega t-q_{2} \sin \omega t\right), \\
x=\mu+\varepsilon\left(q_{1} \sin \omega t+q_{2} \cos \omega t\right), \quad \varepsilon=a L^{2} / \Delta^{2},
\end{gathered}
$$

характеризующего нелинейную структуру электрического поля в области плазменного резонанса. Безразмерные величины $x$ и $\mu$ нормированы на ширину резонанса $\Delta, L$-характерный масштаб неоднородности плотности ионов плазмы. Тот или иной вид функций $q_{1}$ и $q_{2}$ в соотношениях (40) возникает как проявление конкретной зависимости от координаты $x$ электрического поля, являющегося решением линеаризованной полной системы исходных уравнений при соответствующих граничных условиях и с выбранным профилем плотности в области плазменного резонанса.

Например, в случае холодной электронной плазмы с линейным профилем плотности $N(x)$ функции $q_{1}$ и $q_{2}$ имеют вид

$$
q_{1}=\left(1+\mu^{2}\right)^{-1}, \quad q_{2}=\mu\left(1+\mu^{2}\right)^{-1},
$$


причем ширина $\Delta=(\nu / \omega) L$ определяется частотой соударений частиц в плазме.

В случае горячей плазмы, когда сушественно влияние теплового движения электронов, линеаризация исходной системы уравнений дает распределение электрического поля для линейного профиля плотности в области плазменного резонанса в виде функций Эйри-Фока. Соответствующая нелинейная структура электрического поля, плотности и скорости электронов, возникаюшая как продолжение учитывающих первую тепловую поправку линейных соотношений $(a \rightarrow 0)$ с помощью РГ-оператора (38) на область конечных значений параметра $a$, по-прежнему определяется выражениями (40), в которых, однако, ширина резонанса зависит от температуры электронов $\Delta=\left(3 V_{T}^{2} L / \omega^{2}\right)^{1 / 3}$, а функции $q_{i}$ имеют вид

$$
q_{1}=\int_{0}^{\infty} d \xi \cos \left(\mu \xi+\xi^{3} / 3\right), \quad q_{2}=\int_{0}^{\infty} d \xi \sin \left(\mu \xi+\xi^{3} / 3\right) .
$$

Замечательная особенность формул для $v$ и $E$ в (40) с функциями $q_{1}, q_{2}$ из (42) состоит в том, что они дают точное (при $\left.\omega_{L}^{2}=\omega^{2}\right)$ решение уравнений $(36)$, не содержаших вклада электронного давления, и вместе с тем учитывают конечное значение электронной температуры.

Детальный анализ приведенных формул с обсуждением физических следствий результатов содержится в работах $[23,25]$. Здесь мы лишш отметим содержательность результатов, полученных с использованием условия $\Phi$ А для сравнительно простой математической модели даже без учета влияния на РГС отброшенных в нулевом пределе малых поправок, хотя такая модификация РГ-оператора (38) и не представляет особого труда (соответствуюшее выражение для поправок по градиенту плотности указано в [23]). Именно наличие достаточно широкого произвола в виде функций $q_{1}$ и $q_{2}$ в формулах (40) позволяет с единых позиций анализировать нелинейную структуру электрического поля в области плазменного резонанса как в холодной, так и в горячей плазме.

Отметим качественную аналогию РГ-оператора (38) с оператором боголюбовской РГ (4). Как и в классической квантово-полевой РГ, оператор (38) задает преобразования переноса по некоторой характеристике решения - параметру $a$ - и более сложные функциональные преобразования координаты $x$. $\Phi$ А исследуемой физической системы проявляется в инвариантности искомых функций - поля $E$ и скорости электронов $v-$ относительно этих РГ-преобразований. При этом параметр нелинейности $a$, участвующий в РГ-преобразовании, не предполагается малым в отличие от отброшенных при получении оператора $R_{8}$ "приближенной" РГС слагаемых, малость которых использовалась при переходе от исходной системы уравнений к уравнениям (36). Это обстоятельство позволяет использовать РГС для улучшения решения исходных уравнений в виде степенного ряда по параметру а до точного существенно нелинейного решения (40). При этом найденный оператор РГ, как и в квантово-полевой модели, отвечает точному групповому преобразованию по параметру $а$ для решения уравнений (36). Вместе с тем он является приближенным, поскольку получен в условиях малости указанных выше других, отличных от $а$ параметров и допускает последовательное уточнение по этим параметрам.

5.2. ФА в нелинейной волновой оптике. Во многих случаях ограничение лишь нулевыми по малым параметрам вкладами в РГС не представляется возможным. Поэтому ниже рассмотрен вариант РГС (и соответственно условий $\Phi А$ ), который в явном 
виде учитывает вклады в оператор РГ входящих в систему малых параметров. Он возникает при анализе краевой задачи, являющейся обобщением (28), (29),

$$
\begin{gathered}
v_{t}+v v_{x}-\alpha n_{x}-\beta \partial_{x}\left(\left(\frac{x^{1-\nu}}{\sqrt{n}}\right) \partial_{x}\left(x^{\nu-1} \partial_{x}(\sqrt{n})\right)\right)=0 \\
n_{t}+n v_{x}+v n_{x}+(\nu-1) \frac{n v}{x}=0
\end{gathered}
$$

Здесь $\alpha$, как и ранее, - параметр нелинейной рефракции, параметр $\beta$ характеризует интенсивность процесса дифракции, $\nu=1$ и $\nu=2$ отвечают плоской и цилиндрической геометрии пучка. Граничные условия для уравнений (43) определяют на входе в нелинейную среду при $z=0$ кривизну волнового фронта пучка и распределение его интенсивности по поперечной координате $x$ :

$$
v(0, x)=V(x)=-\frac{x}{T}, \quad n(0, x)=N(x) .
$$

5.2.1. Плоская геометрия. Рассмотрим вначале случай плоской геометрии $(\nu=1)$, пренебрежем влиянием дифракции $(\beta=0)$ и ограничимся простым случаем, когда волновой пучок на входе в среду (т.е. при $t=0)$ имеет плоский фазовый фронт. Тогда уравнения (43) сводятся к системе (30). Операторы РГС для краевой задачи (30), (31) запишем в каноническом виде (32). Представим координаты $f$ и $g$ в виде степенных рядов

$$
f=\sum_{i=0}^{\infty} \alpha^{i} f^{i}, \quad g=\sum_{i=0}^{\infty} \alpha^{i} g^{i} .
$$

Способ нахождения коэффициентов $f^{i}$ и $g^{i}$ и конечный результат в виде системы рекуррентных соотношений приведены в работах $[26,27]$. При этом каждый из этих коэффициентов определен с точностью до произвольной функции от $n, \chi_{(s)}$ и комбинации $\tau_{(s)}-w\left(s \chi_{(s)}+n \chi_{(s+1)}\right)$, где $(s)$ означает производную порядка $s$ по $n$. Этот произвол устраняется при сужении группы на искомом решении краевой задачи, т.е. при проверке условия $\Phi$ А (34), которое принимает вид дифференциальной (или алгебраической) связи и при $\tau=0$ согласуется с краевым условием (31). Ограничиваясь конечным числом слагаемых в разложении (45), что возможно при малых значениях $\alpha$, приходим к приближенной симметрии. Последнее означает, что не зависящие от параметра $\alpha$ вклады $f^{0}$ и $g^{0}$ определяются из более простой - по сравнению с исходной $(30)-$ системы уравнений

$$
\tau_{w}-n \chi_{n}=0, \quad \chi_{w}=0 .
$$

В отличие от исходной системы, которая допускает только конечную группу преобразований Ли-Беклунда заданного порядка, эта система допускает бесконечную групп симметрии, характеризуемую произвольной зависимостью координат $f^{0}$ и $g^{0}$ от своих аргументов. Для малых значений $\alpha$ симметрия уравнений (46) наследуется исходной системой (30) в произвольном порядке по этому параметру. Сужение найденной приближенной группы на решении краевой задачи и дает искомую РГС.

Приведем примеры операторов РГС, задаваемых координатами в виде двучленов

$$
f=f^{0}+\alpha f^{1}, \quad g=g^{0}+\alpha g^{1} .
$$


Явньй вид операторов зависит от функций $f^{0}$ и $g^{0}$, которые, в свою очередь, определяются (неоднозначно) формой распределения поперечной интенсивности $N(x)$ на границе нелинейной среды.

Для “солитонного" профиля интенсивности пучка $N(x)=\mathrm{ch}^{-2} x$ можно указать следующие два набора формул для коэффициентов, которые получаются [26] из различных выражений для $f^{0}, g^{0}$ :

$$
\text { a) } \begin{aligned}
& f^{0}=2 n(1-n) \tau_{n n}-n \tau_{n}-2 n w\left(\chi_{n}+n \chi_{n n}\right), \quad f^{1}=\frac{1}{2} n w^{2} \tau_{n n}, \\
& g^{0}=2 n(1-n) \chi_{n n}+(2-3 n) \chi_{n} \\
& g^{1}=w\left(2 n \tau_{n n}+\tau_{n}\right)+\frac{w^{2}}{2}\left(n \chi_{n n}+\chi_{n}\right) ; \\
& \text { б) } f^{0}=1+2 n \chi_{n} \text { th } \chi, \quad f^{1}=\left(\frac{\tau^{2}}{n}-2 \tau \tau_{n}+2 \tau^{2} \text { th } \chi\right) \operatorname{ch}^{-2} \chi, \\
& g^{0}=0, \quad g^{1}=-2 \tau \chi_{n} \operatorname{ch}^{-2} \chi-2 \tau_{n} \text { th } \chi .
\end{aligned}
$$

Как показывают расчеты для вкладов более высокого порядка $[26,28,20]$, в случае "а" функции $f^{i}, g^{i}$ обрашаются в нуль при $i \geqslant 2$ и формулы (48) и (45) описывают точную РГС. Сравнение с координатами (35) показывает, что $f^{1,2}, g^{1,2}$ являются их разложениями по степеням $\alpha$. Для варианта "б" ситуация оказывается иной: ряды (45) не обрываются и формулы (49) и (45) дают приближенную РГС.

Для волнового пучка с начальным профилем интенсивности в виде гауссовой кривой, $N(x)=\exp \left(-x^{2}\right)$, представим два набора $f$ и $g$ операторов приближенной РГС:

$$
\begin{aligned}
& \text { а) } f^{0}=1+2 n \chi \chi_{n}, \quad g^{0}=0, \quad f^{1}=-2 \tau \tau_{n}+\frac{\tau^{2}}{n}, \quad g^{1}=-2\left(\tau \chi_{n}+\chi \tau_{n}\right) \\
& \text { б) } f^{0}=2 n\left(\tau \chi_{n}+\tau_{n} \chi\right), \quad g^{0}=1+2 n \chi \chi_{n}, \quad f^{1}=2 \chi \tau_{\alpha}, \quad g^{1}=2\left(\chi \chi_{\alpha}+\tau \tau_{n}\right)
\end{aligned}
$$

Формулы (48)-(51) демонстрируют достоинство способа построения РГС с использованием приближенной симметрии, позволяющего анализировать краевые задачи с произвольными, вообще говоря, краевыми данными, заданными дифференциальными (либо алгебраическими) выражениями для функций $f^{0}, g^{0}$. При этом пример оператора (48) показывает, что в отдельных случаях подход с использованием приближенных симметрий может приводить к точным РГС и их наличие в анализируемой задаче следует заранее установить с помощью методов, изложенных в предыдущем разделе. Наличие в выражениях (48) для координат $f^{i}$ и $g^{i}$ вторых производных указывает на то, что соответствующий оператор $R$ является оператором РГС Ли-Беклунда второго порядка, в то время как операторы (49)-(51) эквивалентны операторам точечной симметрии. Отметим, что наличие в операторе (51) производной первого порядка по параметру $\alpha$ указывает на то, что преобразования $\Phi$ А вовлекают также параметр нелинейной рефракции [26]. Подробный анализ формул (48)-(51) и физических следствий, возникаюших при подстановке в условия $\Phi$ А $(34)$, дан в работах $[26,22]$.

5.2.2. Цилиндрический случай. Аналогичным способом с использованием условий $\Phi$ А строятся приближенные РГС и для случая $\beta \neq 0$ [27]. В качестве примера укажем 
лишь один оператор РГС для иилиндрического $(\nu=2)$ волнового пучка:

$$
\begin{aligned}
R_{9}= & {\left[\left(1-\frac{t}{T}\right)^{2}+t^{2} S_{\chi \chi}\right] \partial_{t}+\left[-\frac{x}{T}\left(1-\frac{t}{T}\right)+t S_{\chi}+v t^{2} S_{\chi \chi}\right] \partial_{x}+} \\
& +\left[\frac{x}{T^{2}}+\frac{v}{T}\left(1-\frac{t}{T}\right)+S_{\chi}\right] \partial_{v}+ \\
& +\left[\frac{2 n}{T}\left(1-\frac{t}{T}\right)-n t\left(1+\frac{v t}{x}\right) S_{\chi \chi}-\frac{n t}{x} S_{\chi}\right] \partial_{n} .
\end{aligned}
$$

Здесь функция $S$ зависит от переменной $\chi=x-v t$ :

$$
S(\chi)=\alpha N(\chi)+\frac{\beta}{\chi \sqrt{N(\chi)}} \partial_{\chi}\left(\chi \partial_{\chi}(\sqrt{N(\chi)})\right)
$$

содержит два малых параметра $\alpha$ и $\beta$ и определяется видом начального распределения интенсивности пучка $N$ на границе нелинейной среды. При этом, как и при $\beta=0$, существуют такие функции $N$, для которых оператор (52) отвечает не приближенной, а точной РГС, т.е. применимой при произвольных, не малых значениях параметров $\alpha$ и $\beta$ [27]. Преобразования $\Phi$ А возникают из решения уравнений характеристик для сопряженного с (52) ДУ в частных производных первого порядка и дают возможность продолжить решения по ТВ, заданные в малой окрестности границы нелинейной среды, в область, где сушественными становятся сильно нелинейные эффекты [27].

Завершая этот раздел, отметим многообразие форм записи условий $Ф А$ и, как следствие, различные подходы к их дальнейшему использованию, возникающие в рассмотренных задачах с малым параметром. Использование приближенных симметрий приводит к некоторым особенностям в построении РГС и использовании условий $\Phi$ А, которые можно сформулировать следуюшим образом.

Во-первых, это уже обсуждавшийся в предыдушем разделе способ применения условий $\Phi$ А, заданных в виде системы ДУ высокого порядка, когда последние анализируются в совокупности с исходными уравнениями. В отличие от конечных преобразований $\Phi$ А, представленных в этом случае в виде формальных бесконечных рядов $[18$, т. 3 , гл. 1], дифференциальная формулировка условий $\Phi$ А является удобным инструментом для построения решений краевой задачи.

Во-вторых, условия $\Phi$ А в виде приближенных симметрий могут рассматриваться как дополнительные дифференциальные связи, которые в совокупности с исходными уравнениями задают РГ-многообразие. В свою очередь, такое многообразие может быть использовано для построения точечных приближенных РГС.

В-третьих, важность использования условий $\Phi$ А на всех этапах построения приближенных РГС начиная с процедуры отбора координат оператора РГС в невозмушенном решении. Отметим возможность реализации алгоритма построения РГС на основе приближенных симметрий для задач с произвольными краевыми данными, что открывает новые возможности использования метода.

В-четвертых, использование в преобразованиях $\Phi$ А в числе прочих переменных также параметров, которые используются при построении приближенных РГС. 


\section{6. ЗАКЛЮЧЕНИЕ}

Подводя итог, попытаемся сформулировать те основные изменения, которые произошли в понятии $\Phi$ А за последнее десятилетие. Первоначально это понятие сложилось на базе представлений о групповых преобразованиях, развитых в классических работах Софуса Ли [29] и использованных на этапе становления квантово-полевой (боголюбовской) РГ $[4,5]$. В основе этих представлений лежала однопараметрическая группа Ли локальных преобразований, к которой относится и РГ Боголюбова. Введение понятия $\Phi$ А упорядочило представление о различных РГ и дало возможность выявить и сформулировать их единую групповую структуру.

Интенсивное развитие математических методов в исследовании симметрий дифференциальных и интегродифференциальных уравнений, приведших к созданию современного группового анализа (список публикаций на эту тему можно найти в $[10,18,30,31]$, открыло возможность придания нового смысла понятию $\Phi$ А.

В первую очередь это привело к созданию метода построения специального класса симметрий, связанных с РГ-преобразованиями, а именно симметрий ренормгруппового типа или РГС. Наличие такого метода (см. раздел 2) сделало регулярной процедуру нахождения РГ-преобразований, по крайней мере для тех систем, которые описываются с помошью дифференциальных и интегродифференциальных уравнений. Использование инфинитезимального подхода позволило сформулировать условия РГ-инвариантности на языке операторов РГС и употребить для их построения весь арсенал средств современного группового анализа. В результате понятие РГС теперь включает не только точечные симметрии (раздел 3), но и симметрии Ли-Беклунда (высшие или обобшенные симметрии) (разделы 4, 5), приближенные симметрии (раздел 5) и т.д. Далеко не полный список примеров, приведенных в этом обзоре, отвечает наиболее продвинутым результатам в построении РГС, но сформулированный подход позволяет также обсуждать нелокальные РГС и РГС интегродифференциальных уравнений, основываясь, например, на методе, изложенном в [32].

Можно сказать, что современный групповой анализ сыграл в развитии понятия $\Phi$ А ту же роль, что и классический групповой анализ Ли в становлении РГ Боголюбова.

Условия $\Phi$ А формулируются как условия инвариантности относительно преобразований, задаваемых РГ-операторами. Это обстоятельство оказывается важным в том случае, когда возникают проблемы с формулировкой конечных преобразований $\Phi$ А, например для РГС Ли-Беклунда (см. разделы 4, 5). Универсальность этой формулировки оказалась полезной не только на этапе нахождения решений, но и при конструировании операторов РГС. В этом смысле условия $\Phi$ А и проверка их выполнения вошли в метод построения РГС в качестве одного из его составных элементов. При этом сами условия могут принимать вид как алгебраических, так и дифференциальных соотношений, содержащих производные высокого (не только первого, п. 4.2) порядка.

Существенной чертой нового способа нахождения РГС является регулярная возможность построения алгебр Ли конечной размерности. Многочисленные примеры этих алгебр можно найти, например, в работах $[9,19]$, хотя практически они еше не нашли широкого приложения (см. раздел 3). Подобные алгебры Ли отвечают многопараметрическим группам. Аппарат РГС приводит к набору групповых операторов, образующих алгебры Ли.

Использование подобных групп привычно для исследователей, изучающих симмет- 
рию тех или иных уравнений современной теоретической физики"). Однако “ренормализационная группа" как в боголюбовском, т.е. квантово-полевом, так и в вильсоновском вариантах всегда понималась как однопараметрическая группа. Немногочисленные попытки прибегнуть к двухпараметрическим конструкциям всегда, насколько нам известно [33], сводились к прямому произведению двух однопараметрических РГ.

Традиционное назначение РГ-метода в задачах с особенностями - служить инструментом для улучшения ТВ и давать корректное описание поведения решения в окрестности сингулярности - сохранилось и в излагаемом здесь подходе. Именно использование условий $\Phi$, сформулированных в разделах 4 и 5 на базе РГС Ли-Беклунда, позволило описать структуру не только уже известных сингулярных решений, но и тех, которые не были получены ранее (см. [22, 26, 27]). Подчеркнем, что подход с использованием РГС в этих задачах позволил описать структуру двумерных особенностей, отличающихся от особенностей, возникающих в задачах с использованием боголюбовской РГ.

Благодарности. Работа выполнена при частичной поддержке Российского фонда фундаментальных исследований (гранты № 96-15-96030 и № 99-01-00232), а также гранта INTAS № 96-0842.

\section{Список литературы}

[1] Д. В. Ширков. ДАН СССР. 1982. Т. 263. С. 64.

[2] Д. В. Ширков. ТМФ. 1984. Т. 60. С. 218.

[3] D. V. Shirkov. Intern. J. Mod. Phys. A. 1988. V. 3. P. 1321.

[4] E. C. G. Stückelberg, A. Petermann. Helv. Phys. Acta. 1953. V. 26. P. 499.

[5] Н. Н. Боголюбов, Д. В. Ширков. ДАН СССР. 1955. Т. 103. С. 203; С. 391; ЖЭЭТ. 1956. T. 30. C. 77 ; N. N. Bogoliubov, D. V. Shirkov. Nuovo Cimento. 1956. V. 3. P. 845.

[6] K. Wilson. Phys. Rev. B. 1971. V. 4. P. 3184.

[7] Н. Н. Боголюбов, Д. В. Ширков. Введение в теорию квантованных полей. М.: Наука, 3-е изд., 1976; 4-е изд., 1984.

[8] В.З. Бланк, В. Л. Бонч-Бруевич, Д. В. Ширков. ЖЭТФ. 1957. Т. 33. С. 265.

[9] V.F. Kovalev, V. V. Pustovalov, D. V. Shirkov. J. Math. Phys. 1998. V. 39. № 2. P. 1170.

[10] Л. В. Овсянников. Групповой анализ дифференциальных уравнений. М.: Наука, 1978.

[11] L.-Y. Chen, N. Goldenfeld, Y. Oono. Phys. Rev. E. 1996. V. 54. № 1. P. 376.

[12] J. Bricmont, A. Kupiainen, G. Lin,. Commun. Pure Appl. Math. 1994. V. 47. P. 893.

[13] T. Kunihiro. Progr. Theor. Phys. 1995. V. 94. № 4. P. 503.

[14] V.F. Kovalev, S. V. Krivenko, V. V. Pustovalov. The renormalization group method bazed on group analysis. In: Renormalization group-91. Eds. D. V. Shirkov, V.B. Priezhzev. Singapore: World Scientific, 1992. P. 300.

[15] В. Ф. Ковалев, В. В. Пустовалов, Д. В. Ширков. Сообшения ОИЯИ. 1995. № Р5-95-447.

[16] R. M. Corless et al. Adv. Comp. Math. 1996. V. 5. P. 329.

[17] И. Л. Соловцов, Д. В. Ширков. ТМФ. 1999. Т. 120. № 3. С. 482

[18] CRC Handbook of Lie Group Analysis of Differential Equations. Ed. N. H. Ibragimov. V. 1: Symmetries, Exact Solutions and Conservation Laws. Boca Raton, Florida, USA: CRC Press, 1994; V. 2: Applications in Engineering and Physical Sciences, 1995; V.3: New trends in theoretical developments and computational methods, 1996.

[19] V.F. Kovalev, V. V. Pustovalov. Lie Group and their Applications (Istanbul). 1994. V. 1. № 2. P. 104

\footnotetext{
${ }^{3)}$ Например, алгебра группы преобразований Пуанкаре состоит из 10 операторов.
} 
[20] V.F. Kovalev, V. V. Pustovalov. Math. Comput. Modelling. 1997. V. 25. P. 165.

[21] V.F. Kovalev. J. Nonlinear Math. Phys. 1996. V. 3. P. 351.

[22] V. F. Kovalev, D. V. Shirkov. J. Nonlinear Opt. Phys. \& Materials. 1997. V. 6. P. 443.

[23] В. Ф. Ковалев, В. В. Пустовалов. ТМФ. 1989. V. 81. Р. 69.

[24] В.Ф. Ковалев, В.В. Пустовалов. Краткие сообщения по физике ФИАН СССР им. П. Н. Лебедева. 1989. № 3. С. 41.

[25] В. Ф. Ковалев, В. В. Пустовалов. Квантовая электроника. 1988. Т. 15. С. 726; Физика плазмы. 1989. Т. 15. С. 47; С. 563; Квантовая электроника. 1989. Т. 16. С. 2261.

[26] В. Ф. Ковалев. ТМФ. 1997. Т. 111. С. 369.

[27] В. Ф. Ковалев. ТМФ. 1999. Т. 119. № 3. С. 405.

[28] В. Ф. Ковалев, В.В. Пустовалов, С.И. Сенашов. Дифф. уравн. 1993. Т. 29. № 10. C. 1751.

[29] S. Lie. Gesammelte Abhandlungen. Leipzig: B. G. Teubner; Oslo: H. Aschehoug \& Co. Bd. 1, 1934; Bd. 2 (Teil 1), 1935; Bd. 2 (Teil 2), 1937; Bd. 3, 1922; Bd. 4, 1929; Bd. 5, 1924; Bd. 6, 1927.

[30] Peter J. Olver. Applications of Lie Groups to Differential Equations. N. Y.: Springer-Verlag, 1986.

[31] Симметрии и законы сохранения уравнений математической физики. Ред. А.М. Виноградов, И.С. Красильщик. М.: Факториал, 1997.

[32] В.Ф. Ковалев, С. В. Кривенко, В. В. Пустовалов. Дифф. уравн. 1993. Т. 29. С. 1804; C. 1971.

[33] C. Stephens. Int. J. Mod. Phys. 1998. V. 12. P. 1379; Mod. Phys. Lett. A. 1994. V. 9. P. 309. 